M. Dehghani, M. Mardaneh, Z. Montazeri, A. Ehsanifar, M.J. Ebadi, O.M. Grechko

\title{
SPRING SEARCH ALGORITHM FOR SIMULTANEOUS PLACEMENT OF DISTRIBUTED GENERATION AND CAPACITORS
}

\begin{abstract}
Purpose. In this paper, for simultaneous placement of distributed generation (DG) and capacitors, a new approach based on Spring Search Algorithm (SSA), is presented. This method is contained two stages using two sensitive index Sv and Ss. Sv and Ss are calculated according to nominal voltage and network losses. In the first stage, candidate buses are determined for installation $D G$ and capacitors according to $S v$ and $S s$, Then in the second stage, placement and sizing of distributed generation and capacitors are specified using SSA. The spring search algorithm is among the optimization algorithms developed by the idea of laws of nature and the search factors are a set of objects. The proposed algorithm is tested on 33-bus and 69-bus radial distribution networks. The test results indicate good performance of the proposed method. References 30, tables 4, figures 4 . Key words: DG placement, capacitor placement, distribution network, SSA, sensitive index, two-stage simultaneous placement.
\end{abstract}

\begin{abstract}
Цель. В статье для одновременного размещения распределенной генерации и конденсаторов представлен новый подход, основанный на «пружинном» алгоритме поиска (Spring Search Algorithm, SSA). Данный метод состоит из двух этапов $c$ использованием двух показателей чувствительности Sv и Ss. Показатели чувствительности Sv и Ss рассчитываются в соответствии с номинальным напряэением и потерями в сети. На первом этапе определяются шины-кандидаты для установки распределенной генерации и конденсаторов согласно Sv и Ss. Затем, на втором этапе размещение и калибровка распределенной генерации и конденсаторов выполняются с использованием алгоритма SSA. «Пружинный» алгоритм поиска входит в число алгоритмов оптимизации, разработанных на основе идей законов природы, а факторы поиска представляют собой набор объектов. Предлагаемый алгоритм тестируется на радиальных распределительных сетях с 33 и 69 шинами. Результаты тестирования показывают хорошую эффективность предложсенного метода. Библ. 30, табл. 4, рис. 4 .

Ключевые слова: размещение распределенной генерации, размещение конденсаторов, распределительная сеть, «пружинный» алгоритм поиска SSA, показатель чувствительности, двухэтапное одновременное размещение.
\end{abstract}

Introduction. Lately, electricity trading and connecting distributed generation (DG) to the distribution network has been placed under private investors' scope of interest. Besides, placing capacitators within medium voltage networks is a paramount factor, which is noticed highly by distribution companies. Noticeably since the DG and capacitor are related to each other as sources of active and reactive power, performing their placement at the same time makes more optimal solution found.

Placement of DGs. The distribution system planning requires DGs to be placed properly within the distribution system. In definition, DG known as a small generator is responsible of generating Stand Alone and On Grid electricity [1].

In placing DGs, some methods can be used. [2, 3] refers to the sizing and analytical method by which DGs can be placed and sized properly. The objective function of the mentioned reference is minimizing the loss. In [4], DGs are placed by considering some objective functions known as increasing the spinning reserve, improving the voltage profile, decreasing the load flow and decreasing the transmission loss. In [5], based on the fuzzy logic the algorithm known as Bellman-zadeh is used for DGs placement. Reference [6], uses the load flow method by applying the voltage profile and the power loss such that it computes some objective function optimal paramount factors first, then decreases the transmission loss and finally improves the voltage profile. The method of DGs placement in [7], is based on the voltage stability analysis known as a security measure. It is proper to mention that energy efficiency can be improved by applying two strategies known as conservation voltage reduction (CVR) and DG integration. In [8], CVR and DG placement are studied to find their interaction in minimizing the load consumption of distribution networks. It is noted that the afore-mentioned process is performed by keeping the lowest voltage level within the predefined range. [9], refers highly to the economic and network-driven DG placement planning. Its viewpoint is from the local distribution company (DISCO) considering reliability level and the electrical distribution network power loss.

Placement of Capacitor. Capacitators have long been applied within the industrial plants and commercial establishments for the purpose of a power factor (PF) improvement. Besides, electric utilities use capacitator to control the feeder voltage and to improve the distribution system efficiency. Based on studies conducted in recent decades [10-22], there are different models and mathematical solution techniques for the capacitator placement. More elaborately, Schmill [19], applied a uniform characteristics feeder comprising a uniform load distribution. He used the two-thirds rule that is he used a single capacitor along with the two-thirds of the feeder length. In [12], dynamic programming (DP) was used by Duran to arrive at the optimal solution.

However, the application of a uniform load and a fixed conductor size was abolished by Grainger and Lee [16], who searched to find the optimal solution by dividing the problem into three sub problems known as size, switching time, and location. Noticeably, these phases were successively solved. In [18], load data were gathered from the distribution feeder by Rembert and Rinker who used a reactive current recorder to compute the instantaneous apparent and reactive currents. Moreover, in order to find the optimal solution Sundhararajan [21], applied a directed grid search method to decrease the number of candidate nodes such that by rejecting other nodes, the top two or three nodes in each 
lateral branch were merely used. Xu [22], in his method of capacitor banks placement could reduce the power loss. Indeed, the low-side of transformers was considered a proper location for the capacitor bank. Some other important factors were taken into account by Kaur [15], like cost, size and location of capacitor bank. Ultimately, he could compensate the reactive power demand by using the load. An integrated optimization method enjoying a sequential strategy and multi objectives was proposed and then applied by $\mathrm{Su}[20]$, who could optimally place and then control the delta-connected switched capacitors.

Simultaneous placement of DGs and Capacitors. A more optimal solution can be achieved if DGs and capacitators are placed properly at the same time. This ideal motivated many researchers to seek its performing procedure such that in [23], this ideal was sought within the radial distribution network with different load levels. The same investigation was done in [24], In this study through the simultaneous placement of DG and capacitor in a radial distribution network, researchers aimed to arrive at the optimal quantity, placement, and sizing. To fulfil this aim, researchers chose a new manner as the multi objective optimization problem, which encompassed the DG units', and capacitors' costs, power losses, and voltage stability margins. This problem performing process was to apply a developed genetic algorithm as the first stage in the proposed hierarchical optimization strategy. The other type of simultaneous capacitor banks and distributed generation allocation comprising the stochastic nature of DG was done in [25], in which the Tabu -search and genetic algorithms-based efficient hybrid method was proposed.

Likewise, in the present paper, a new method is suggested to simultaneously allocate DGs and Capacitors in distribution systems. Accordingly, concerning the nominal voltage of buses and network losses, two new sensitivity indexes are introduced. The first index is called the sensitivity index of voltage $(S v)$ which is based on the buses nominal voltage. The second one is the sensitivity index of losses network, which is related to the network losses. The present methodology operates within two stages; in the first one, candidate buses are chosen to install DG and capacitators concerning both sensitivity indexes of $S v$ and $S s$; though, in the second stage, DG and capacitators placement and sizing are done by applying SSA. It is proper to mention that, the present study is indeed a case study using IEEE 33-bus of [7] and 69-bus of [25] and results are reported as well.

Problem Formulation. Minimizing the overall system loss and improving the system voltage profile are indeed two principal objectives of DGs optimal sizing and placement. Noticeably, in this process the bus and branch number must be minutely adjusted to find the answer of the load flow distribution (Fig. 1).

The process of branch numbering is as follows:

- choosing the root, swing or the slack bus when the main source is connected;

- ordering layers from the first layer of tree in which all branches connected to root bus;

- labelling the second layer of tree, which is connected to the first layer receiving end bus and so on.

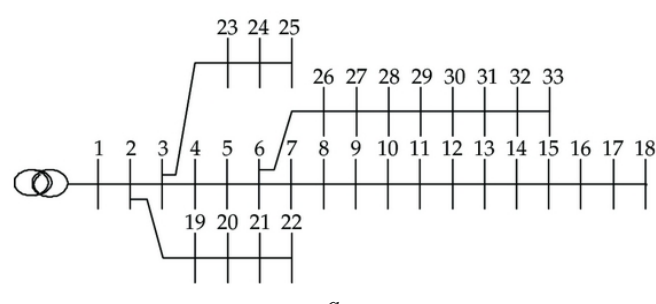

$a$

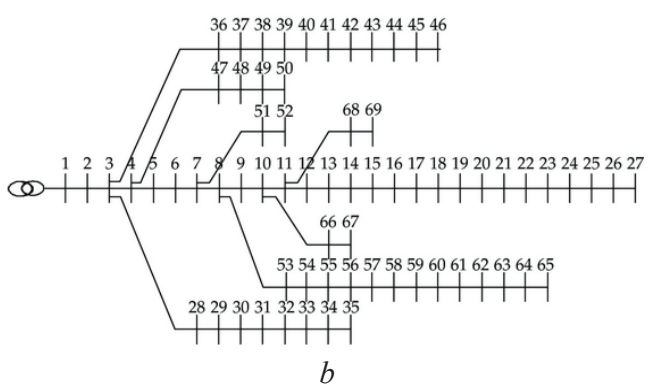

Fig.1. IEEE 33-bus distribution network (a); IEEE 69-bus distribution network $(b)$

In an assumed $\Pi$ model of the network in which I branch is attached to $k$ bus in one hand and to the $m$ bus on the other hand, $k$ bus is closer to the root bus that is the net power goes from $k$ to $m$ bus. Fig. 2 represents the power flow through the series impedance of the branch. These flows are represented in (1) and (2), elaborately.

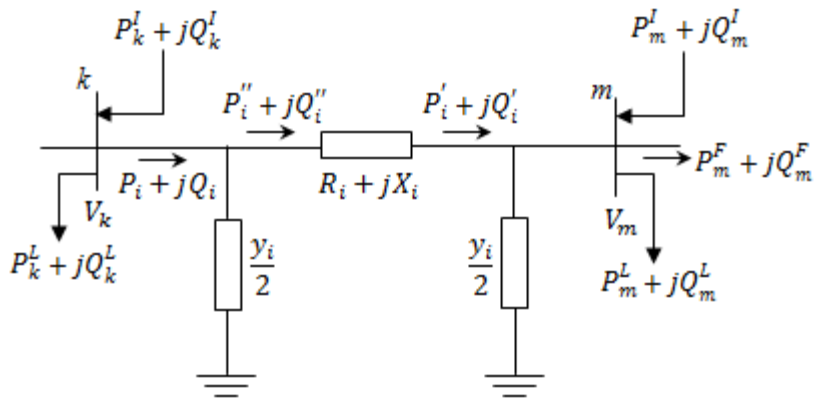

Fig. 2. П model of a network

$$
\begin{gathered}
P_{i}^{\prime}=P_{m}^{L}+P_{m}^{F}-P_{m}^{I}, \\
Q_{i}^{\prime}=Q_{m}^{L}+Q_{m}^{F}-Q_{m}^{I}-V_{m}^{2} \frac{y_{i}}{2},
\end{gathered}
$$

where $L, F$ and $I$ subscripts represents the load, the flow and the injection respectively. Branch $i$ let the power flow near bus $k$. This passage can be formulated as

$$
\begin{gathered}
P_{i}=P_{i}^{\prime \prime}=P_{i}^{\prime}+R_{i} \frac{P_{i}^{\prime 2}+Q_{i}^{\prime 2}}{V_{m}^{2}}, \\
Q_{i}=Q_{i}^{\prime \prime}-V_{k}^{2} \frac{y_{i}}{2}=Q_{i}^{\prime}+X_{i} \frac{P_{i}^{\prime 2}+Q_{i}^{\prime 2}}{V_{m}^{2}}-V_{k}^{2} \frac{Y_{i}}{2},
\end{gathered}
$$

In order to calculate the power flow quantity in each branch of tree, it is computed recursively in a backward/anti clock-wise direction. Thus, the bus $m$ complex voltage is computed as

$$
V_{m}=\left[V_{k}-\frac{P_{i}^{\prime \prime} R_{i}+Q_{i}^{\prime \prime} X_{i}}{V_{k}^{*}}\right]-j\left[\frac{P_{i}^{\prime \prime} X_{i}-Q_{i}^{\prime \prime} R_{i}}{V_{k}^{*}}\right] .
$$

The strategy of finding the magnitude and angle of all buses voltages of the tree is to compute this complex voltage in a forward direction. 
This computation is done iteratively again and again till the voltage difference at loop breaking points (breaking points of the tree) is placed within the acceptable limit. Hence the branch $I$ active power loss $\left(P_{L i}\right)$ and reactive power loss $\left(Q_{L i}\right)$ are measured as

$$
\begin{gathered}
P_{L i}=P_{i}^{\prime \prime}-P_{i}^{\prime}=R_{i} \frac{P_{i}^{\prime 2}+Q_{i}^{\prime 2}}{V_{m}^{2}}, \\
Q_{L i}=Q_{i}^{\prime \prime}-Q_{i}^{\prime}=X_{i} \frac{P_{i}^{\prime 2}+Q_{i}^{\prime 2}}{V_{m}^{2}} .
\end{gathered}
$$

Accordingly, quantities of the system net active, reactive and apparent power loss are

$$
\begin{gathered}
P_{L}=\sum_{i=1}^{N} P_{L i} ; \\
Q_{L}=\sum_{i=1}^{N} Q_{L i} ; \\
S_{L}=\left(P_{L}^{2}+Q_{L}^{2}\right)^{\frac{1}{2}},
\end{gathered}
$$

where $S_{L}$ is the distribution system apparent power loss.

As mentioned, the major objective of the present problem is to minimize the net power loss and modify the voltage profile of the system. It is proper to re-mention that the present paper seeks some other minor objectives like optimal sizing and placement of DGs and capacitators.

SSA or the spring search Algorithm optimization technique is known as a robust and a very few user dependent parameter which has a very good convergence characteristic. Besides, it doesn't stagnate at the local minima. This characteristic is the major reason of selecting SSA to minimize the system the net power loss and to modify the voltage profile.

Methodology. In this study, stimulatory placement and sizing of DG and Capacitors are determined in two stages base on two sensitivity indexes.

Sensitivity indexes $S v$ and $S s$ are defined as

$$
\begin{gathered}
S v^{j}=\sum_{i=1}^{N B}\left|V_{i}-1\right|, \\
S s^{j}=\frac{S_{L}^{i}}{S_{L}^{b}},
\end{gathered}
$$

where $S v^{j}$ is the sensitivity index of voltage in perchance Capacitor in Bus $j, N B$ is the number of buses, $S s^{j}$ is the sensitivity index of loss power in perchance DG in bus $j$, $S_{L}^{i}$ is the apparent power loss in perchance DG in bus $j$, and $S_{L}^{b}$ is the apparent power loss in base case.

First stage. DGs and capacitors must be installed at the appropriate position. This position must have an acceptable impact on the characteristics of the network. So it is important to identify a suitable location for installing DGs and capacitors. In the first stage, candidate locations are determined. For this purpose $S v$ and $S v$ for all buses are calculated. Then, priorities of each bus for installing DG and capacitor are identified according to the $S v$ and $S s$.

Second stage. In the first stage, priorities of buses are determined. Prioritization of Buses makes the search space reduces and also increase accuracy. Now size and place of DGs and capacitors should be determined. For this purpose SSA is used. The objective function introduces as

$$
\begin{gathered}
\text { objective function }=k_{1} S s^{n}+k_{2} S v^{n}, \\
\qquad k_{1}+k_{2}=1,
\end{gathered}
$$

where $k_{1}$ and $k_{2}$ is the weight factors, $S v^{n}$ and $S s^{n}$ are the normalized sensitivity indexes.

The Spring Force Optimization [26, 27]. Optimization algorithms have been used in many applications in electrical engineering [28] such as operation of electrical energy [29] and energy management [30].

The SSA algorithm is explained in two phases: 1 - making an artificial system with the discrete time in the problem atmosphere, the initial positioning of objects, determining the governing laws and principles, and arranging parameters, 2 - passing the time until arriving at the stop time.

Forming system, determining the laws, and adjustment of the parameter. In the first step, the system atmosphere is determined. This atmosphere includes a multi- dimensional coordinates within the problem definition atmosphere. Any point in the space is the answer to the problem. Searching factors are a set of objects, which are attached to each other by springs. Indeed, each object is attached to any other objects by means of spring, and each object has the characteristics of the springs' position and stiffness coefficient attached to it. The object position is a point in the space where it is a solution of the problem. The springs' stiffness coefficients can be determined concerning the suitability of any two objects attached to one another.

After making the system, its governing laws are determined. It is supposed that the governing laws are merely the spring law and the motion law. The general schematic of these laws are approximately similar to the nature laws and it is defined as below:

The spring force law (Hook law). In physics, mechanics and the elastic material science, the spring force law is an approximate which shows that a material length change has a linear relationship with its imposed force. Most materials follow this rule with a good (acceptable) approximate till the imposed force is lower than their elastic ability. Any deviation from the Hook law can be increased by increasing deformations such that in many deformations when the material trespasses the linear elastic domain, the Hook law loses its applicability $[23,24]$. In the present article, it is supposed that the Hook law is always satisfied.

The motion laws. The present movement of each object equals to the coefficient sum of the object last position and its dislocation. Any object dislocation can be determined concerning the spring force law [23].

Now assume the system as a set of $m$ objects. The position of each object is a point in the space where it can be the answer to the optimization problem. In equation (15), the $d$ position of $I$ object is shown with $x_{i}^{d}$

$$
X_{i}=\left(x_{i}^{1}, \ldots, x_{i}^{d}, \ldots, x_{i}^{n}\right) .
$$


At first, the objects position is defined within the problem definition atmosphere randomly. These objects pave the way to arrive at the balance point (solution) concerning forces imposed to each other by spring.

In order to compute the spring stiffness, equation (16) is used

$$
K_{i, j}=K_{\max }\left|F_{n}^{i}-F_{n}^{j}\right| \max \left(F_{n}^{i}, F_{n}^{j}\right),
$$

where $K_{i, j}$ is the spring stiffness between $i$ and $j$ objects, $K_{\max }$ represents the maximum quantity of the spring stiffness which is determined regarding the problem type, $F_{n}$ shows the normalised objective function, and $F_{n}^{i}, F_{n}^{j}$ are the normalised objective functions of $i$ and $j$ objects.

In order to normalise the objective function, equations (17) and (18) are used:

$$
\begin{gathered}
F_{n}^{\prime i}=\frac{f_{o b j}^{i}}{\min \left(f_{o b j}\right)} ; \\
F_{n}^{i}=\min \left(F_{n}^{\prime i}\right) \times \frac{1}{F_{n}^{\prime i}},
\end{gathered}
$$

where $f_{o b j}$ is the objective function and $f_{o b j}^{i}$ is the objective function quantity of object $i$.

In a problem with $m$ variables, it is possible to suppose that the problem has $m$ dimensions; hence, it is possible to define a coordinate for each dimension; thus, it is plausible to depict the equivalent of any system variable on the related coordinate. On each coordinate, the robust/strong points of the right side and left side of the object are determined concerning the comparison of the objective function quantity. The robust/strong points of each object are indeed those objects, which are in fact in a more optimal position, rather than the object itself. Therefore, on each coordinate, two total sum forces are imposed to the object: the right side sum forces and the left side sum forces. In order to compute these forces, there are

$$
\begin{aligned}
& F_{\text {total }_{R}}^{j, d}=\sum_{i=1}^{n_{R}^{d}} K_{i, j} x_{i, j}^{d} ; \\
& F_{\text {total }_{L}}^{j, d}=\sum_{l=1}^{n_{L}^{d}} K_{l, j} x_{l, j}^{d},
\end{aligned}
$$

where, respectively, $F_{\text {total }_{R}}^{j, d}$ and $F_{\text {total }_{L}}^{j, d}$ are the resultant force imposed to object $j$ from the right and left side at the dimension $d ; n_{R}^{d}$ and $n_{L}^{d}$ are respectively the number of right and left robust/strong points of $d$ th dimension; $K_{i, j}$ and $K_{l, j}$ show the spring stiffness connected to $j$ object on one hand and the strong points on the other hand.

Now by applying the Hook law in $d$ th dimension, there are

$$
\begin{aligned}
& d X_{R}^{j, d}=\frac{F_{\text {total }_{R}}^{j, d}}{K_{\text {equal }_{R}}^{j}} ; \\
& d X_{L}^{j, d}=\frac{F_{\text {total }_{L}}^{j, d}}{K_{\text {equal }_{L}}^{j}},
\end{aligned}
$$

where $d X_{R}^{j, d}$ and $d X_{L}^{j, d}$ are respectively the $j$ object dislocation to the right and to the left in the $d$ th dimension.

Therefore, there is

$$
d X^{j, d}=d X_{R}^{j, d}+d X_{L}^{j, d},
$$

where $d X^{j, d}$ is the $j$ object ultimate dislocation along with the $d$ dimension.

This dislocation quantity can be positive or negative concerning equation (23). Now, there is

$$
X^{j, d}=X_{0}^{j, d}+r_{1} \times d X^{j, d},
$$

where $X^{j, d}$ is related to the new balance point place and time of the $j$ object in the $d$ dimension; $X_{0}^{j, d}$ is the $j$ object initial balance point along with the $d$ dimension. Here, there is a random number with a constant distribution within $[0,1]$ time span which is used to keep the random mode of the search.

The passing of time and the parameter updating. At the beginning of the system formation, any object is randomly placed in a point in the space where it is the answer to the problem. At each moment of the time, objects are assessed and then their dislocations are computed after calculating equations (16) to (23). At the later time, the object holds a place at that position. The present used parameter is the spring stiffness coefficient which is updated at each level based on equation (16). The stop point can be determined after passing a definite time. The spring force algorithm different steps are shown as below:

1. Determining the system atmosphere and the allocating the initial quantities;

2. The initial positioning of objects;

3. Assessing and normalising the objects suitability;

4. The $k$ parameter updating;

5. Forming the spring force and motion laws for each object;

6. Computing the values of objects dislocations;

7. Updating the objects positions/locations;

8. Repeating steps 3 to 7 till the stop point is satisfied;

9. Ending.

Simulation result. In order to simulate the proposed problem, the IEEE 33-bus and IEEE 69-bus radial network is used. The networks data, including the resistance and reactance of the lines and the loads connected to nodes, were presented in $[7,25]$. In order to show the importance of studying the simultaneous placement and sizing of the DG units and the capacitors, first, for the proposed networks, placement, and sizes of the DG units and the capacitors are presented separately, and finally, the simultaneous placement and sizing of the DGs and capacitors is determined and the results are compared.

Placement and sizing of DG. In this section, placement and sizing of DG units regarding the minimum value of the problem objective function are defined. The results for this case are shown in Table 1.

Placement and sizing of Capacitor. In this section, placement and sizing of capacitors regarding the minimum value of the problem objective function are defined. The results for this case are shown in Table 2. 
Table 1 buses for installation DG and capacitors has been

Placement and sizing of DGs

\begin{tabular}{|c|c|c|}
\hline Network & Capacity (kW) & Bus No. \\
\hline \multirow{3}{*}{$33-b u s$} & 1987.1971 & 14 \\
\cline { 2 - 3 } & 656.4803 & 30 \\
\cline { 2 - 3 } & 203.0234 & 31 \\
\hline \multirow{3}{*}{ 69-bus } & 958.3215 & 60 \\
\cline { 2 - 3 } & 245.2561 & 61 \\
\cline { 2 - 3 } & 260.4957 & 62 \\
\hline
\end{tabular}

Table 2

\begin{tabular}{|c|c|c|}
\multicolumn{3}{|c}{ Placement and sizing of Capacitors } \\
\hline Network & Capacity (kVAr) & Bus No. \\
\hline \multirow{2}{*}{ 33-bus } & 551.1281 & 14 \\
\cline { 2 - 3 } & 986.8425 & 30 \\
\hline \multirow{2}{*}{ 69-bus } & 746.4401 & 64 \\
\cline { 2 - 3 } & 654.3891 & 65 \\
\hline
\end{tabular}

Simultaneous placement and sizing of DG and Capacitors. In this section, placement and sizing of DG units and capacitors regarding the minimum value of the problem objective function are defined. The results for this case are shown in Table 3.

Table 3

Placement and sizing of DGs and capacitors

\begin{tabular}{|c|c|c|c|c|}
\hline Network & Capacity (kW) & Bus No. & Capacity (kVAr) & Bus No. \\
\hline \multirow{3}{*}{ 33-bus } & 504.8423 & 14 & 892.5297 & 12 \\
\cline { 2 - 5 } & 489.5621 & 16 & 344.2624 & 30 \\
\cline { 2 - 5 } & 750.3225 & 32 & - & - \\
\hline \multirow{3}{*}{ 69-bus } & 893.2986 & 60 & 786.5941 & 24 \\
\cline { 2 - 5 } & 221.2428 & 61 & 702.4423 & 25 \\
\cline { 2 - 5 } & 857.5422 & 62 & - & - \\
\hline
\end{tabular}

Losses and $S v$ of the system different case of study are shown in Table 4. The voltages profile of network before and after the installation of the DGs and capacitors are shown in Fig. 3 and Fig. 4.

Losses and $S v$ of the network

\begin{tabular}{|c|c|c|c|}
\hline Network & Case study & Power losses (kVA) & $S v$ \\
\hline \multirow{3}{*}{ IEEE } & Base & 243.6003 & 1.7009 \\
\cline { 2 - 4 } 33-Bus & DG & 106.5249 & 0.5301 \\
\cline { 2 - 4 } & CAPACITOR & 155.1845 & 0.7368 \\
\cline { 2 - 4 } & DG and CAPACITOR & 78.6582 & 0.2162 \\
\hline \multirow{3}{*}{ IEEE } & Base & 247.0873 & 1.8367 \\
\cline { 2 - 4 } 69-Bus & DG & 83.7836 & 0.5513 \\
\cline { 2 - 4 } & CAP & 158.5726 & 0.7068 \\
\cline { 2 - 4 } & DG and CAPACITOR & 29.6673 & 0.1278 \\
\hline
\end{tabular}

Discussion. According to the above simulation results, the operation from the network that is in the presence of DG provided better conditions than the operation in the presence of the capacitors, which shows that the role of DG is more effective than that of the capacitors. Also, the simulation results show that the optimal operation of the network occurs in the simultaneous expansion planning of DGs and capacitors. Comparing the results, it is obvious that optimal operation from the network is obtained by the simultaneous placement of DG units and capacitors.

Conclusions. In this paper, we have presented a new approach for simultaneous placement and sizing of distributed generation and capacitors. Two sensitive indexes has been defined based on voltage profile and power loss of the network. Proposed methodology has been implemented in two stages. In the first stage, the candidate determined. Then in the second stage, the places and sizes of the DGs and Capacitors have been located using SSA. The proposed algorithm is tested on IEEE-33 bus and IEEE-69 bus distribution system. The voltage profile and power loss of the system has improved to acceptable limit.

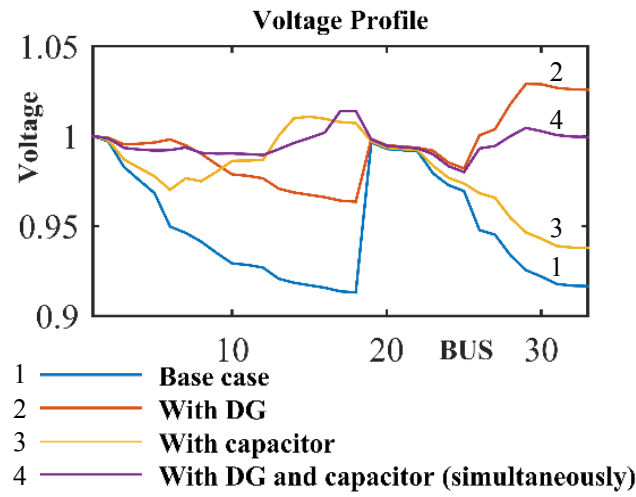

Fig. 3. Voltage profile of IEEE 33-Bus

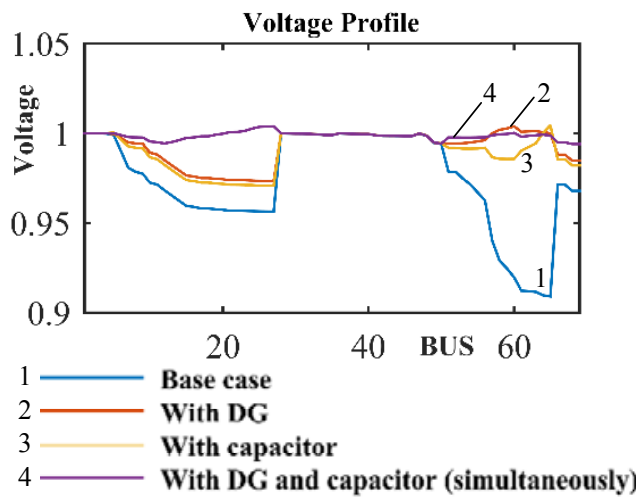

Fig. 4. Voltage profile of IEEE 69-Bus

\section{REFERENCES}

1. Daly P.A. Morrison J. Understanding the potential benefits of distributed generation on power delivery systems. 2001 Rural Electric Power Conference. Papers Presented at the 45th Annual Conference (Cat. No.01CH37214). doi: 10.1109/repcon.2001.949510.

2. Gözel T., Hocaoglu M.H. An analytical method for the sizing and siting of distributed generators in radial systems. Electric Power Systems Research, 2009, vol.79, no.6, pp. 912918. doi: 10.1016/j.epsr.2008.12.007.

3. Wang C., Nehrir M.H. Analytical approaches for optimal placement of distributed generation sources in power systems. IEEE Transactions on Power Systems, 2004, vol.19, no.4, pp. 2068-2076. doi: 10.1109/tpwrs.2004.836189.

4. Abou El-Ela A.A., Allam S.M., Shatla M.M. Maximal optimal benefits of distributed generation using genetic algorithms. Electric Power Systems Research, 2010, vol.80, no.7, pp. 869-877. doi: 10.1016/j.epsr.2009.12.021.

5. Barin A., Pozzatti L.F., Canha L.N., Machado R.Q., Abaide A.R., Arend G. Multi-objective analysis of impacts of distributed generation placement on the operational characteristics of networks for distribution system planning. International Journal of Electrical Power \& Energy Systems, 2010, vol.32, no.10, pp. 1157-1164. doi: 10.1016/j.ijepes.2010.06.015.

6. Ghosh S., Ghoshal S.P., Ghosh S. Optimal sizing and placement of distributed generation in a network system. International Journal of Electrical Power \& Energy Systems, 2010, vol.32, no.8, pp. 849-856. doi: 10.1016/j.ijepes.2010.01.029.

7. Ettehadi M., Ghasemi H., Vaez-Zadeh S. Voltage stabilitybased DG placement in distribution networks. IEEE Transactions on Power Delivery, 2013, vol.28, no.1, pp. 171-178. doi: 10.1109/tpwrd.2012.2214241.

8. Wang Z., Chen B., Wang J., Begovic M.M. Stochastic DG placement for conservation voltage reduction based on multiple 
replications procedure. IEEE Transactions on Power Delivery, 2015, vol.30, no.3, pp. 1039-1047. doi: 10.1109/tpwrd.2014.2331275. 9. Rahmani-andebili M. Distributed generation placement planning modeling feeder's failure rate and customer's load type. IEEE Transactions on Industrial Electronics, 2016, vol.63, no.3, pp. 1598-1606. doi: 10.1109/tie.2015.2498902.

10. Baran M.E., Wu F.F. Optimal capacitor placement on radial distribution systems. IEEE Transactions on Power Delivery, 1989, vol.4, no.1, pp. 725-734. doi: 10.1109/61.19265.

11. Chiang H.-D., Wang J.-C., Cockings O., Shin H.-D. Optimal capacitor placements in distribution systems. I. A new formulation and the overall problem. IEEE Transactions on Power Delivery, 1990, vol.5, no.2, pp. 634-642. doi: 10.1109/61.53065.

12. Dura H. Optimum number, location, and size of shunt capacitors in radial distribution feeders a dynamic programming approach. IEEE Transactions on Power Apparatus and Systems, 1968, vol.PAS-87, no.9, pp. 1769-1774. doi: 10.1109/tpas.1968.291982.

13. Fawzi T., El-sobki S., Abdel-halim M. New approach for the application of shunt capacitors to the primary distribution feeders. IEEE Transactions on Power Apparatus and Systems, 1983 vol.PAS-102, no.1, pp. 10-13. doi: 10.1109/tpas.1983.317991.

14. Kaplan M. Optimization of number, location, size, control type, and control setting of shunt capacitors on radial distribution feeders. IEEE Transactions on Power Apparatus and Systems, 1984, vol.PAS-103, no.9, pp. 2659-2665. doi: $10.1109 /$ tpas.1984.318238.

15. Kaur H., Kumar P., Sharma A., Kamaiya N. A study on optimal capacitor placement in distribution system: Conventional and Artificial Intelligence techniques. 2015 International Conference on Computation of Power, Energy, Information and Communication (ICCPEIC), Apr. 2015. doi: 10.1109/iccpeic.2015.7259457.

16. Lee S., Grainger J. Optimum placement of fixed and switched capacitors on primary distribution feeders. IEEE Transactions on Power Apparatus and Systems, 1981, vol.PAS100 , no.1, pp. $345-352$. doi: $\mathbf{1 0 . 1 1 0 9 / t p a s . 1 9 8 1 . 3 1 6 8 6 2}$.

17. Ponnavsikko M., Rao K.S. Optimal choice of fixed and switched shunt capacitors on radial distributors by the method of local variations. IEEE Transactions on Power Apparatus and Systems, 1983, vol.PAS-102, no.6, pp. 1607-1615. doi: 10.1109/tpas.1983.317890.

18. Rinker R.E., Rembert D.L. Using the reactive current profile of a feeder to determine optimal capacitor placement. IEEE Transactions on Power Delivery, 1988, vol.3, no.1, pp. 411-416. doi: $10.1109 / 61.4272$.

19. Schmill J.V. Optimum size and location of shunt capacitors on distribution feeders. IEEE Transactions on Power Apparatus and Systems, 1965, vol.84, no.9, pp. 825-832. doi: 10.1109/tpas.1965.4766262

20. Su X., Masoum M.A.S., Wolfs P.J. PSO and improved BSFS based sequential comprehensive placement and real-time multi-objective control of delta-connected switched capacitors in unbalanced radial $\mathrm{MV}$ distribution networks. IEEE Transactions on Power Systems, 2016, vol.31, no.1, pp. 612 622. doi: 10.1109/tpwrs.2015.2398361.

21. Sundhararajan S., Pahwa A. Optimal selection of capacitors for radial distribution systems using a genetic algorithm. IEEE Transactions on Power Systems, 1994, vol.9, no.3, pp. 14991507. doi: 10.1109/59.336111.

22. Xu Y., Dong Z.Y., Wong K.P., Liu E., Yue B. Optimal capacitor placement to distribution transformers for power loss reduction in radial distribution systems. IEEE Transactions on Power Systems, 2013, vol.28, no.4, pp. 4072-4079. doi: 10.1109/tpwrs.2013.2273502.
23. Sajjadi S.M., Haghifam M.-R., Salehi J. Simultaneous placement of distributed generation and capacitors in distribution networks considering voltage stability index. International Journal of Electrical Power \& Energy Systems, 2013, vol.46, pp. 366-375. doi: 10.1016/j.ijepes.2012.10.027.

24. Esmaeilian H.R., Darijany O., Mohammadian M. Optimal placement and sizing of DG units and capacitors simultaneously in radial distribution networks based on the voltage stability security margin. Turkish Journal of Electrical Engineering \& Computer Science, 2014, p. 1-14. doi: 10.3906/elk-1203-7.

25. Pereira B.R., Martins da Costa G.R.M., Contreras J., Mantovani J.R.S. Optimal distributed generation and reactive power allocation in electrical distribution systems. IEEE Transactions on Sustainable Energy, 2016, vol.7, no.3, pp. 975984. doi: 10.1109/tste.2015.2512819.

26. Dehghani M., Montazeri Z., Dehghani A., Seifi A.R. Spring search algorithm: A new meta-heuristic optimization algorithm inspired by Hooke's law. 2017 IEEE 4th International Conference on Knowledge-Based Engineering and Innovation (KBEI). doi: 10.1109/kbei.2017.8324975.

27. Dehghani M., Montazeri Z., Dehghani A., Nouri N., Seifi A. BSSA: Binary spring search algorithm. 2017 IEEE 4th International Conference on Knowledge-Based Engineering and Innovation (KBEI), Dec. 2017., pp. 0220-0224. doi: 10.1109/kbei.2017.8324977

28. Dehghani M., Montazeri Z., Ehsanifar A., Seifi A.R., Ebadi M.J., Grechko O.M. Planning of energy carriers based on final energy consumption using dynamic programming and particle swarm optimization. Electrical engineering \& electromechanics, 2018, no.5, pp. 62-71. doi: 10.20998/2074-272X.2018.5.10.

29. Montazeri Z., Niknam T. Optimal utilization of electrical energy from power plants based on final energy consumption using gravitational search algorithm. Electrical engineering \& electromechanics, 2018, no.4, pp. 70-73. doi: 10.20998/2074272X.2018.4.12.

30. Montazeri Z., Niknam T. Energy carriers management based on energy consumption. 2017 IEEE 4th International Conference on Knowledge-Based Engineering and Innovation (KBEI), Dec. 2017. doi: 10.1109/kbei.2017.8325036.

Received 14.07.2018

M. Dehghani ${ }^{1}$, Candidate of Power Engineering, PhD Student,

M. Mardaneh ${ }^{1}$, Doctor of Power Engineering, Associate

Professor,

Z. Montazeri ${ }^{2}$, Candidate of Power Engineering, M.Sc.,

A. Ehsanifar ${ }^{3}$, Candidate of Power Engineering, M.Sc.,

M.J. Ebadi ${ }^{4}$, Doctor of Applied Mathematics, Assistant

Professor,

O.M. Grechko ${ }^{5}$, Candidate of Technical Science, Associate

Professor,

${ }^{1}$ Department of Electrical and Electronics Engineering,

Shiraz University of Technology, Shiraz, Iran,

e-mail: adanbax@gmail.com, mardaneh@sutech.ac.ir

${ }^{2}$ Department of Electrical Engineering,

Islamic Azad University of Marvdasht, Marvdasht, Iran,

e-mail: Z.montazeri2002@gmail.com

${ }^{3}$ Department of Power and Control,

Shiraz University, Shiraz, Iran,

e-mail: ali.ehsanifar2020@gmail.com

${ }^{4}$ Faculty of Marine Science,

Chabahar Maritime University, Chabahar, Iran,

e-mail: ebadi@cmu.ac.ir

${ }^{5}$ National Technical University «Kharkiv Polytechnic Institute»,

2, Kyrpychova Str., Kharkiv, 61002, Ukraine,

e-mail: a.m.grechko@gmail.com

How to cite this article:

Dehghani M., Mardaneh M., Montazeri Z., Ehsanifar A., Ebadi M.J., Grechko O.M. Spring search algorithm for simultaneous placement of distributed generation and capacitors. Electrical engineering \& electromechanics, 2018, no.6, pp. 68-73. doi: 10.20998/2074-272X.2018.6.10. 